\title{
Resistant Hypertension and Obstructive Sleep Apnea: The Sparring Partners
}

\section{Costas Thomopoulos, ${ }^{1}$ Helena Michalopoulou, ${ }^{2}$ Alexandros Kasiakogias, ${ }^{3}$ Anna Kefala, ${ }^{3}$ and Thomas Makris ${ }^{1}$}

\author{
${ }^{1}$ Department of Cardiology, Elena Venizelou Hospital, 2 Elena Venizelou Sqr., Athens 11521, Greece \\ ${ }^{2}$ Department of Cardiology, Metaxa Cancer Hospital, 51 Mpotassi Str., Piraeus 18537, Greece \\ ${ }^{3}$ First Cardiology Clinic, University of Athens, Hippokration Hospital, 114 Vas. Sofias Str., Athens 11527, Greece
}

Correspondence should be addressed to Costas Thomopoulos, thokos@otenet.gr

Received 14 October 2010; Accepted 8 December 2010

Academic Editor: Vasilios Papademetriou

Copyright (c) 2011 Costas Thomopoulos et al. This is an open access article distributed under the Creative Commons Attribution License, which permits unrestricted use, distribution, and reproduction in any medium, provided the original work is properly cited.

\begin{abstract}
Enhanced target organ damage and cardiovascular morbidity represent common issues observed in both resistant hypertension and obstructive sleep apnea. Common pathophysiological features and risk factors justify their coexistence, especially in individuals with increased upper-body adiposity. Impaired sodium handling, sympathetic activation, accelerated arterial stiffening, and impaired cardiorenal hemodynamics contribute to drug-resistant hypertension development in obstructive sleep apnea. Effective CPAP therapy qualifies as an effective "add-on" to the underlying antihypertensive pharmacological therapy, and emerging evidence underlines the favorable effect of mineralocorticoid antagonists on both resistant hypertension and obstructive sleep apnea treatment.
\end{abstract}

\section{Introduction}

In the recent years several original articles and review papers have focused on the association between arterial hypertension and obstructive sleep apnea syndrome (OSA) [1-4]. Such an association is supported by the interplay of common risk factors and pathophysiological pathways affecting both the vascular wall and the upper airway [5]. Regarding risk factors, the obesity epidemic has prompted the recognition of an interaction between OSA and increased blood pressure (BP) [6]; additionally, as the population ages and gains weight, resistant hypertension is becoming more prevalent [7].

Up to date few small epidemiological studies have shown a significant association between resistant hypertension and OSA, and based on that both the Seventh Report of the Joint National Committee and the 2007 European Society of Cardiology-European Society of Hypertension guidelines for the management of arterial hypertension recognized OSA as an identifiable and not uncommon cause of resistant hypertension $[8,9]$. Surprisingly, the European guidelines added
OSA to the causes of secondary hypertension, thus creating some confusion; indeed, it is yet unresolved whether OSA could represent just a bystander of essential hypertension and whether the gold standard therapeutic approach for OSA, namely, continuous positive airway pressure (CPAP), is accompanied by efficacious treatment of hypertension $[4,5]$. Either way, misdiagnosed or inappropriately treated OSA may prevent pharmacological treatment of drug-resistant essential hypertension from reducing BP levels within the goal [10].

In the present paper, we illustrate the pathophysiological and epidemiological evidence that supports the interrelationship between resistant hypertension and OSA and how these observations could be translated in clinical decisions in the context of an outpatient hypertension clinic.

\section{Pathophysiological Issues}

2.1. Obesity. Subjects with increased body size are more likely to suffer from hypertension and exhibit difficultto-control hypertension compared to nonobese controls 
[11-13]. Increased free leptin is at least partly associated with increased central and peripheral sympathetic firing in experimental animal models $[14,15]$, while nonapneic and nonobese subjects with out-of-office hypertension are characterized by hyperleptinemia with respect to their normotensive counterparts [16]. Therefore, it is plausible that leptin could represent an accelerator of sympathetic activation in both obesity and hypertension alone. Obesity itself is frequently observed in OSA patients in whom sympathetic activation is further augmented by episodic nocturnal hypoxia and hypercapnia due to the repetitive collapse of the upper vulnerable airway, yet whether leptin levels are raised in normotensive nonobese patients with OSA is still debated [17]. Additionally, sympathetic overactivity per se is accompanied by BP and heart rate increases, as well as by enhanced sodium retention. The above operating mechanisms in obese hypertensive patients with OSA are at least partly responsible for the increased hemodynamic load, and frequently three or more drugs in the maximum tolerated doses are insufficient to effectively reduce BP levels.

2.2. Kidney Function. Focusing on the kidney in obese subjects, "pressure natriuresis" is impaired, and higher BP levels are required to achieve sodium balance [18]. Additionally, urinary albumin excretion is increased in obese OSA patients with hypertension compared to hypertensive nonOSA controls [19]. A further hypothetical mechanism by which OSA affects renal hemodynamics is that the continuous nocturnal intrathoracic pressure swings are accompanied by changes in systemic and renal venous pressure, as it has been shown that renal venous congestion is accompanied by a decline in kidney function in patients with both preserved and impaired cardiac function undergoing right heart catheterization [20]. Considering the above, kidney impairment in OSA subjects may contribute to the development of drugresistant hypertension, and diuretics implementation may be accompanied by better antihypertensive results by promoting both sodium excretion and venous decongestion.

2.3. Aldosterone. Plasma aldosterone levels are increased in both obese hypertensive patients and those with OSA [6]. Renin-angiotensin-aldosterone axis activation is implicated in most hypertensive states, and apart from sympathetic overdrive, visceral adiposity could also participate in that phenomenon by mechanisms that have not yet been fully elucidated [21]. In patients with resistant hypertension who were referred to an outpatient hypertensive unit, those with a high clinical suspicion for OSA exhibited increased plasma aldosterone levels with respect to those with no symptoms of OSA. In those with a final positive diagnosis of OSA as well as in obese hypertensive subjects, renin activity was suppressed, a finding that suggests that aldosterone secretion is at least partly plasma renin independent; thus, in resistant hypertensive patients with OSA targeting of renin inhibition seems pathophysiologically a less justified therapeutic strategy compared to blockers of subsequent steps of the reninangiotensin-aldosterone cascade. Such a concept is further supported by the finding that hyperaldosteronism prevalence in resistant hypertension is as high as $20 \%$, with obesity and suspected OSA being the two extremely common clinical features in this setting $[21,22]$.

2.4. Arterial Stiffening. Arterial stiffening constitutes a pathophysiological substrate that promotes acceleration of vascular aging observed in both hypertension and OSA [23]. Increased levels of circulating vasoconstrictors [24], enhanced subclinical inflammation [25], endothelial dysfunction [25], and repetitive increases of left ventricle afterload may separately or in combination contribute to the increasing magnitude of arterial stiffening. Even though it is known that resistant hypertension is more prevalent in the elderly, increased arterial stiffness observed in OSA may also precipitate the development of the drug-resistance phenomenon in younger ages.

\section{Epidemiological Issues}

3.1. Noninterventional Studies. In the beginning of the present millennium Logan et al. [26] demonstrated for the first time in the literature the striking prevalence $(82.9 \%)$ of OSA in 41 middle-aged obese patients with "true drugresistant hypertension" recruited from a hospital university hypertension clinic. Although most men with resistant hypertension suffered from OSA (95.8\%), among women those not affected by the syndrome $(35.3 \%)$ were younger, received less drugs, presented with a longer sleep time by $30 \%$ and less arousal movements per hour of sleep by $80 \%$, and finally demonstrated a higher 24-hour pulse pressure, compared to those not affected by OSA. Another surprising finding in the same study was that almost one third of OSA patients with resistant hypertension had a normal dipping profile, suggesting that the nondipping phenomenon in resistant hypertension may be driven by OSA-independent pathophysiological mechanisms.

In another cross-sectional case-control study from the same investigational group [27], patients with drug-resistant hypertension were compared with a group of patients with controlled hypertension matched for age, sex, and body mass index. Accordingly, Ruttanaumpawan et al. [27] reported that those with controlled hypertension-as expectedreceived fewer antihypertensive medications and the use of diuretics was $60 \%$ less frequent with respect to those with resistant hypertension. Moreover, the prevalence of OSA was almost 50\% more frequent in those with resistant hypertension. Total time asleep, sleep efficiency, and time of rapid-eye movement sleep were significantly higher in those with controlled hypertension compared to their resistant hypertension counterparts; interestingly, only the two latter parameters in tandem with the presence of OSA were unadjusted determinants of resistant hypertension in the total study population. After adjusting for confounders, OSA and reduced time of rapid-eye movement sleep qualified as independent significant determinants of resistant hypertension. Among antihypertensive medications, calcium blockers implementation was associated with a lower amount of rapid-eye movement sleep, suggesting that disruption of sleep architecture might be associated in some degree with the selection of antihypertensive drugs. 
In a case-control study, Gonçalves et al. [28] demonstrated that patients with resistant hypertension are affected almost 5 times more frequently by OSA-diagnosed by portable sleep monitors-compared to their well-controlled hypertensive counterparts matched for age, sex, and body mass index. Finally, in a Spanish article by Martínez-García et al. [29], the severity of OSA diagnosed by polysomnography as measured by the apnea/hypopnea index was associated with the magnitude of BP levels in elderly patients with difficult-to-control hypertension and OSA after adjustment for age and sex.

Another study reported that, among resistant hypertension patients, there is a positive association between plasma aldosterone levels and severity of OSA. However, no correlation was noted between OSA and aldosterone levels in subjects without resistant hypertension with equally severe OSA [30]. These findings confirm the high prevalence of OSA in resistant hypertension (85\%) and are consistent with the hypothesis that hyperaldosteronism in resistant hypertension might exacerbate OSA - by promoting upper airway edema due to sodium and fluid retention-or inversely, OSA stimulates aldosterone release, while there might even be a bidirectional relationship in that phenomenon.

3.2. Interventional Studies. Logan et al. [31] tested the therapeutic impact of CPAP therapy on ambulatory BP levels in 11 patients with drug-resistant hypertension and OSA both at a single night's application and after a period of two months. CPAP efficiently applied for one night was accompanied by a significant reduction in nocturnal BP levels. More specifically, nocturnal systolic BP exhibited a more pronounced acute reduction compared to a more limited reduction of the nocturnal diastolic component $(138.3 \pm 6.8$ to $126.0 \pm 6.3 \mathrm{mmHg}$ versus $77.7 \pm 4.5$ to $72.9 \pm 4.5 \mathrm{mmHg}$, resp.). Additionally, CPAP use for 2 months was accompanied by an $11.0 \pm 4.4 \mathrm{mmHg}$ decrease in 24-hour systolic BP, whereas the nocturnal diastolic BP was reduced significantly by $7.8 \pm 3.0 \mathrm{mmHg}$. In these lines, efficiently treating OSA in resistant hypertension might be a therapeutic "add-on" option to the underlying antihypertensive treatment to reduce BP levels within the goal.

In a subsequent work on 33 elderly patients with difficultto-treat hypertension and OSA [32], Martínez-García et al. highlighted, along with the beneficial effect of CPAP on systolic ambulatory BP levels, the fact that compliance to CPAP therapy ( $>4$ hours at night on CPAP) is crucial for the efficient reduction of BP. Indeed, those who tolerated CPAP $(n=23)$ showed a significant drop in mean 24-hour systolic BP of $7.6 \mathrm{mmHg}$ without significant changes in mean 24hour diastolic BP; however, in those who were CPAP intolerant $(n=10)$, there were no significant changes in either mean diurnal or nocturnal, either systolic or diastolic BP.

Lozano et al. [33] in a randomized prospective controlled study evaluated the change in mean 24-hour systolic and diastolic BP at the three-month follow-up in two groups of patients with resistant hypertension and OSA: 29 patients under well-tolerated CPAP and appropriate pharmacological treatment (i.e., CPAP arm) and 35 patients under appropriate pharmacological treatment alone (i.e., conventional arm). The use of CPAP for three months was accompanied by a mean reduction of almost $5 \mathrm{mmHg}$ in 24hour diastolic BP in the CPAP arm, whereas no significant change was observed in the arm of conventional therapy. In those wearing the mask for at least 5.8 hours per day significant changes were registered in diurnal and 24-hour diastolic BP and in 24-hour systolic BP. It is worth noting that the beneficial effect of CPAP on BP levels was only seen in those with resistant hypertension confirmed with ambulatory BP monitoring, but not in those with resistant hypertension diagnosed only by office BP measurements. The number of patients in the CPAP arm with a nondipping pattern decreased significantly from $51.7 \%$ at baseline to $24.1 \%$ at the three-month follow-up, whereas no changes were observed in patients of the conventional arm of the study, in line with previous findings. A final important issue in the Lozano et al. [33] study was that, among those who accepted to wear the mask, the Epworth Sleepiness scale scoring did not differ between those who used CPAP for more and less than 5.8 hours/day, suggesting that even asymptomatic patients with resistant hypertension had similar reductions in BP levels especially if CPAP was applied for more than the cut-off of 5.8 hours/day.

In a retrospective chart review study [34], medical records of patients who had a polysomnography with CPAP study were reviewed and among them the researchers selected those with hypertension; OSA compliant with CPAP; office BP measurements obtained within three months of enrollment, every three months for six months and at one year after CPAP initiation. In subjects exhibiting resistant hypertension, the use of CPAP therapy was accompanied by a decrease in daytime BP at both 6 and 12 months after treatment initiation, while in almost $70 \%$ of these patients a reduction in either the dose or the number of antihypertensive drugs used at baseline was observed. On the contrary, patients with controlled hypertension and OSA demonstrated no significant change in BP levels over the same period of time, suggesting that the effect of CPAP on BP, when the latter has been already controlled with medication, is at least less pronounced or even absent in line with previous findings $[35,36]$.

\section{Clinical Decisions: Lost in Translation or a Simple Rationale?}

In patients with hypertension a thorough sleep history confirmed - if applicable - by the bed partner, identification of daytime symptoms, and a physical examination including visualization of the pharynx are all essential steps in order to separate those with a high clinical suspicion of OSA. However, in patients with drug-resistant hypertension establishing a high or low clinical suspicion for OSA might be futile. For example, in the context of resistant hypertension, the use of clinical prediction tools, like the Epworth Sleepiness Scale could not be as helpful [33] as it may be for the general population of hypertensive patients [4]. Therefore, in clinical practice, those with true drug-resistant hypertension should be counseled to undergo polysomnography. 
Along with the other diagnostic exams, in true drugresistant hypertension, a 24-hour ambulatory BP monitoring should be considered valuable as it confirms the office evaluation of BP levels and possibly helps the clinician assess the hemodynamic responsiveness to CPAP treatment better [33]. Furthermore, evaluating the dipping pattern and nighttime BP levels in resistant hypertension is vital for a more comprehensive approach of the circadian BP variability, its response to therapy, and the potential implementation of chronotherapy issues [4].

Lifestyle modifications should always stand at the first line of the therapeutic procedure in resistant hypertension associated with OSA. The importance of appropriate weight control must be underlined, and bariatric surgery should be encouraged if morbid obesity coexists. Additionally, the underlying pathophysiological mechanisms activated in the duo of resistant hypertension and OSA prompt to rigorous sodium intake restriction [4].

In resistant hypertension complicated by OSA, CPAP is imperative as it proved an efficacious "add-on" to the conventional pharmacological treatment and succeeded to reverse nondipping profile in most of the patients [31, 33]. In these lines, patients should be encouraged to wear their mask every night and possibly for the whole night. The antihypertensive effect of CPAP seems to be more prominent in those with more severe OSA or hypertension, though there is limited evidence whether sleepy and nonsleepy patients with drug resistant hypertension benefit the same in terms of $\mathrm{BP}$ reduction when compliant to CPAP treatment. Although, there is limited clinical evidence for the potential benefit of mineralocorticoid antagonists in resistant hypertension and OSA [37], their use should be considered as an additional promising therapeutic strategy with a plausible pathophysiological background $[6,21]$.

Since resistant hypertension and OSA are accompanied either separately or in combination with highly increased prevalence of target organ damage and adverse cardiovascular outcome, combined treatment seems to be of the highest clinical priority. Targeting on both the maximum individual doses of antihypertensive drugs and the effective implementation of CPAP should remain the main awareness of the physician dealing with this compound difficult-totreat hypertensive disorder.

\section{References}

[1] P. Lavie, P. Herer, and V. Hoffstein, "Obstructive sleep apnoea syndrome as a risk factor for hypertension: population study," British Medical Journal, vol. 320, no. 7233, pp. 479-482, 2000.

[2] F. Javier Nieto, T. B. Young, B. K. Lind et al., "Association of sleep-disordered breathing sleep apnea, and hypertension in a large community-based study," Journal of the American Medical Association, vol. 283, no. 14, pp. 1829-1836, 2000.

[3] O. Friedman and A. G. Logan, "The price of obstructive sleep apnea-hypopnea: hypertension and other ill effects," American Journal of Hypertension, vol. 22, no. 5, pp. 474-483, 2009.

[4] C. Tsioufis, A. Kasiakogias, C. Thomopoulos, A. Manolis, and C. Stefanadis, "Managing hypertension in obstructive sleep apnea: the interplay of continuous positive airway pressure, medication and chronotherapy," Journal of Hypertension, vol. 28, no. 5, pp. 875-882, 2010.

[5] C. Tsioufis, C. Thomopoulos, and C. Stefanadis, "Obstructive sleep apnoea and hypertension: a growing clinical challenge," Hellenic Journal of Cardiology, vol. 49, no. 5, pp. 299-302, 2008.

[6] T. L. Goodfriend, "Obesity, sleep apnea, aldosterone, and hypertension," Current Hypertension Reports, vol. 10, no. 3, pp. 222-226, 2008.

[7] R. J. Garrison, W. B. Kannel, J. Stokes, and W. P. Castelli, "Incidence and precursors of hypertension in young adults: the Framingham offspring study," Preventive Medicine, vol. 16, no. 2, pp. 235-251, 1987.

[8] A. V. Chobanian, G. L. Bakris, H. R. Black et al., "The seventh report of the Joint National Committee on prevention, detection, evaluation, and treatment of high blood pressure: the JNC 7 report," Journal of the American Medical Association, vol. 289, no. 19, pp. 2560-2572, 2003.

[9] G. Mancia, G. De Backer, A. Dominiczak et al., "2007 ESH-ESC practice guidelines for the management of arterial hypertension: ESH-ESC task force on the management of arterial hypertension," Journal of Hypertension, vol. 25, no. 9, pp. 1751-1762, 2007.

[10] O. Marrone, C. Lombardi, and G. Parati, "Effects of continuous positive airway pressure therapy on hypertension control in patients with sleep-related breathing disorders: available evidence and unresolved issues," Journal of Hypertension, vol. 28, no. 10, pp. 2012-2015, 2010.

[11] K. M. Rexrode, J. E. Manson, and C. H. Hennekens, "Obesity and cardiovascular disease," Current Opinion in Cardiology, vol. 11, no. 5, pp. 490-495, 1996.

[12] P. Bramlage, D. Pittrow, H. U. Wittchen et al., "Hypertension in overweight and obese primary care patients is highly prevalent and poorly controlled," American Journal of Hypertension, vol. 17, no. 10, pp. 904-910, 2004.

[13] W. C. Cushman, C. E. Ford, J. A. Cutler et al., "Success and predictors of blood pressure control in diverse North American settings: te antihypertensive and lipid-lowering treatment to prevent heart attact trial (ALLHAT)," Journal of Clinical Hypertension, vol. 4, no. 6, pp. 393-404, 2002.

[14] W. G. Haynes, "Role of leptin in obesity-related hypertension," Experimental Physiology, vol. 90, no. 5, pp. 683-688, 2005.

[15] N. Tümer, B. Erdös, M. Matheny, I. Cudykier, and P. J. Scarpace, "Leptin antagonist reverses hypertension caused by leptin overexpression, but fails to normalize obesity-related hypertension," Journal of Hypertension, vol. 25, no. 12, pp. 2471-2478, 2007.

[16] C. Thomopoulos, D. P. Papadopoulos, O. Papazachou et al., "Free leptin is associated with masked hypertension in nonobese subjects a cross-sectional study," Hypertension, vol. 53, no. 6, pp. 965-972, 2009.

[17] H. Schäfer, D. Pauleit, T. Sudhop, I. Gouni-Berthold, S. Ewig, and H. K. Berthold, "Body fat distribution, serum leptin, and cardiovascular risk factors in men with obstructive sleep apnea," Chest, vol. 122, no. 3, pp. 829-839, 2002.

[18] J. E. Hall, "The kidney, hypertension, and obesity," Hypertension, vol. 41, no. 3, pp. 625-633, 2003.

[19] C. Tsioufis, C. Thomopoulos, K. Dimitriadis et al., "Association of obstructive sleep apnea with urinary albumin excretion in essential hypertension: a cross-sectional study," American Journal of Kidney Diseases, vol. 52, no. 2, pp. 285-293, 2008.

[20] K. Damman, V. M. van Deursen, G. Navis, A. A. Voors, D. J. van Veldhuisen, and H. L. Hillege, "Increased central venous pressure is associated with impaired renal function and 
mortality in a broad spectrum of patients with cardiovascular disease," Journal of the American College of Cardiology, vol. 53, no. 7, pp. 582-588, 2009.

[21] T. L. Goodfriend and D. A. Calhoun, "Resistant hypertension, obesity, sleep apnea, and aldosterone: theory and therapy," Hypertension, vol. 43, no. 3, pp. 518-524, 2004.

[22] D. A. Calhoun, M. K. Nishizaka, M. A. Zaman, and S. M. Harding, "Aldosterone excretion among subjects with resistant hypertension and symptoms of sleep apnea," Chest, vol. 125, no. 1, pp. 112-117, 2004.

[23] C. Tsioufis, K. Thomopoulos, K. Dimitriadis et al., "The incremental effect of obstructive sleep apnoea syndrome on arterial stiffness in newly diagnosed essential hypertensive subjects," Journal of Hypertension, vol. 25, no. 1, pp. 141-146, 2007.

[24] J. E. Dimsdale, T. Coy, M. G. Ziegler, S. Ancoli-Israel, and J. Clausen, "The effect of sleep apnea on plasma and urinary catecholamines," Sleep, vol. 18, no. 5, pp. 377-381, 1995.

[25] C. Thomopoulos, C. Tsioufis, K. Dimitriadis et al., "Obstructive sleep apnoea syndrome is associated with enhanced sub-clinical inflammation and asymmetric dimethyl-arginine levels in hypertensives," Journal of Human Hypertension, vol. 23, no. 1, pp. 65-67, 2009.

[26] A. G. Logan, S. M. Perlikowski, A. Mente et al., "High prevalence of unrecognized sleep apnoea in drug-resistant hypertension," Journal of Hypertension, vol. 19, no. 12, pp. 2271-2277, 2001.

[27] P. Ruttanaumpawan, C. Nopmaneejumruslers, A. G. Logan, A. Lazarescu, I. Qian, and T. D. Bradley, "Association between refractory hypertension and obstructive sleep apnea," Journal of Hypertension, vol. 27, no. 7, pp. 1439-1445, 2009.

[28] S. C. Gonçalves, D. Martinez, M. Gus et al., "Obstructive sleep apnea and resistant hypertension: a case-control study," Chest, vol. 132, no. 6, pp. 1858-1862, 2007.

[29] M. A. Martínez-García, R. Gómez-Aldaraví, T. Gil-Martínez, J. J. Soler-Cataluña, B. Bernácer-Alpera, and P. Román-Sánchez, "Sleep-disordered breathing in patients with difficult-tocontrol hypertension," Archivos de Bronconeumologia, vol. 42, no. 1, pp. 14-20, 2006.

[30] M. N. Pratt-Ubunama, M. K. Nishizaka, R. L. Boedefeld, S. S. Cofield, S. M. Harding, and D. A. Calhoun, "Plasma aldosterone is related to severity of obstructive sleep apnea in subjects with resistant hypertension," Chest, vol. 131, no. 2, pp. 453-459, 2007.

[31] A. G. Logan, R. Tkacova, S. M. Perlikowski et al., "Refractory hypertension and sleep apnoea: effect of CPAP on blood pressure and baroreflex," European Respiratory Journal, vol. 21, no. 2, pp. 241-247, 2003.

[32] M. A. Martínez-García, R. Gómez-Aldaraví, J. J. SolerCataluña, T. G. Martínez, B. Bernácer-Alpera, and P. RománSánchez, "Positive effect of CPAP treatment on the control of difficult-to-treat hypertension," European Respiratory Journal, vol. 29, no. 5, pp. 951-957, 2007.

[33] L. Lozano, J. L. Tovar, G. Sampol et al., "Continuous positive airway pressure treatment in sleep apnea patients with resistant hypertension: a randomized, controlled trial," Journal of Hypertension, vol. 28, pp. 2161-2168, 2010.

[34] T. A. Dernaika, G. T. Kinasewitz, and M. M. Tawk, "Effects of nocturnal continuous positive airway pressure therapy in patients with resistant hypertension and obstructive sleep apnea," Journal of Clinical Sleep Medicine, vol. 5, no. 2, pp. 103107, 2009.

[35] F. Campos-Rodriguez, A. Grilo-Reina, J. Perez-Ronchel et al., "Effect of continuous positive airway pressure on ambulatory
BP in patients with sleep apnea and hypertension: a placebocontrolled trial," Chest, vol. 129, no. 6, pp. 1459-1467, 2006.

[36] F. Campos-Rodriguez, J. Perez-Ronchel, A. Grilo-Reina, J. Lima-Alvarez, M. A. Benitez, and C. Almeida-Gonzalez, "Long-term effect of continuous positive airway pressure on BP in patients with hypertension and sleep apnea," Chest, vol. 132, no. 6, pp. 1847-1852, 2007.

[37] K. Gaddam, E. Pimenta, S. J. Thomas et al., "Spironolactone reduces severity of obstructive sleep apnoea in patients with resistant hypertension: a preliminary report," Journal of Human Hypertension, vol. 24, pp. 532-537, 2009. 


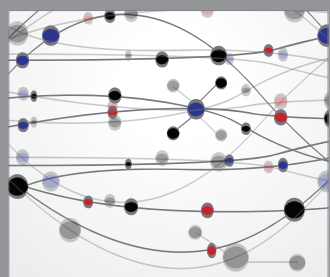

The Scientific World Journal
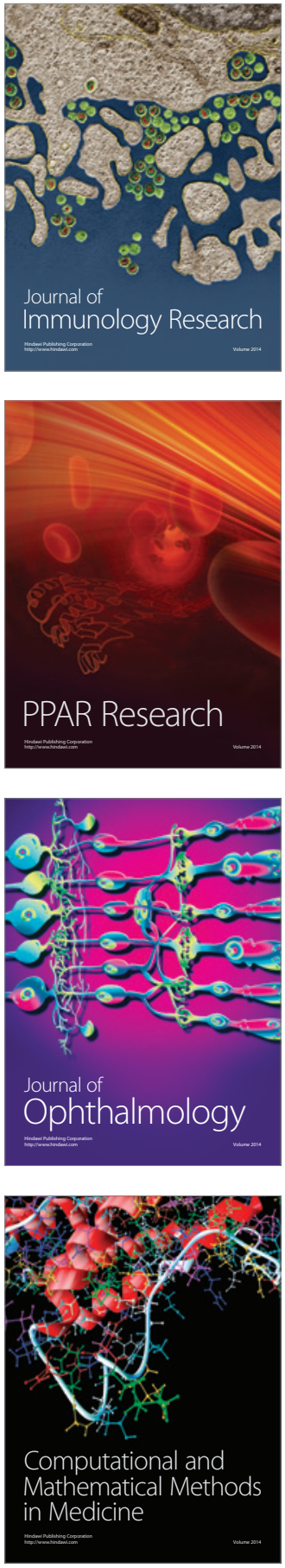

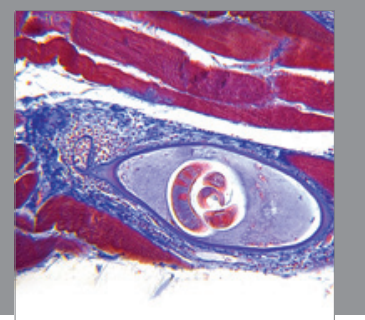

Gastroenterology

Research and Practice
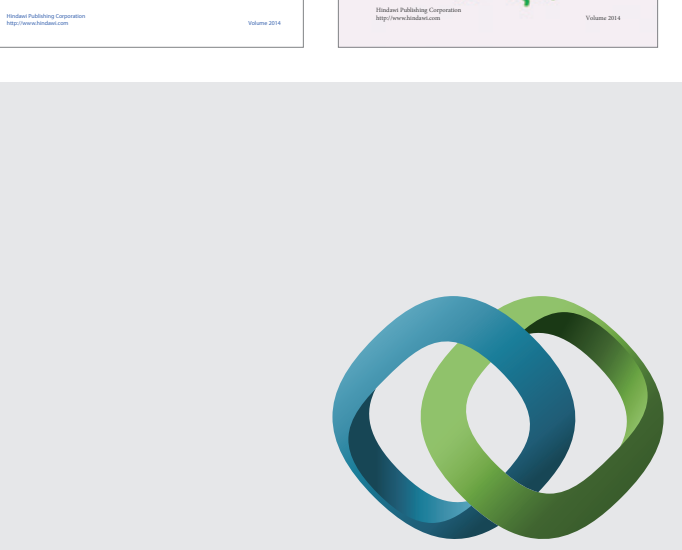

\section{Hindawi}

Submit your manuscripts at

http://www.hindawi.com
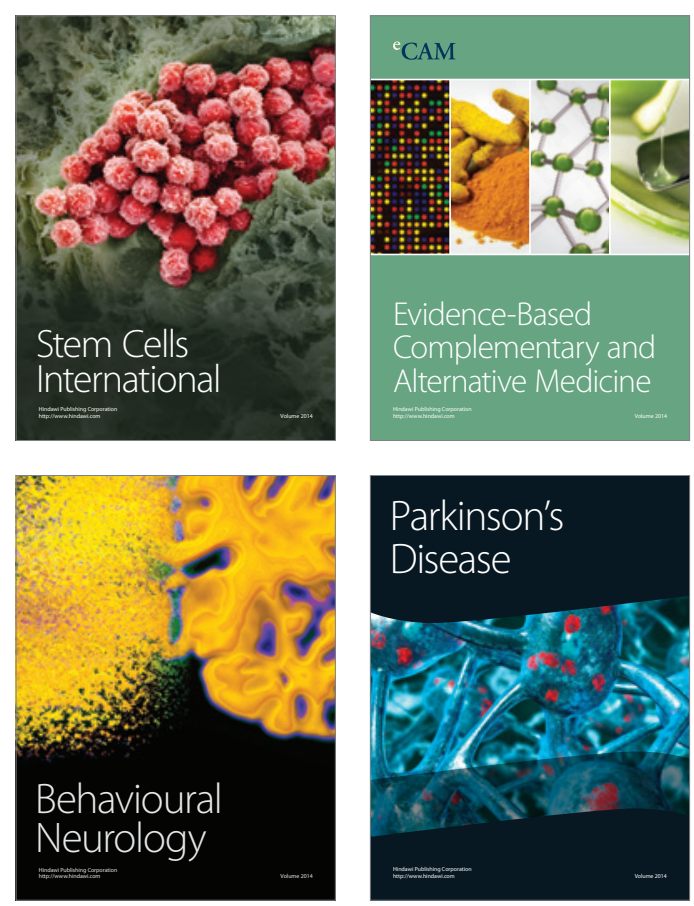

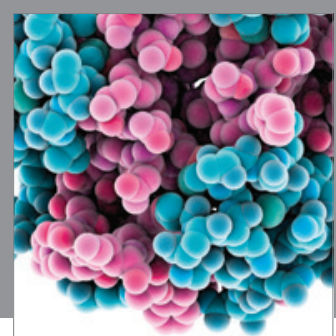

Journal of
Diabetes Research

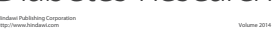

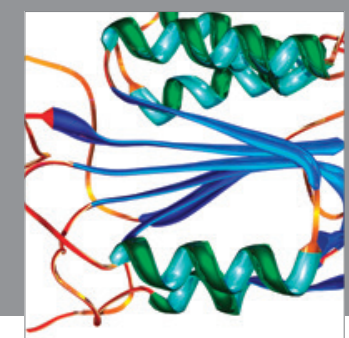

Disease Markers
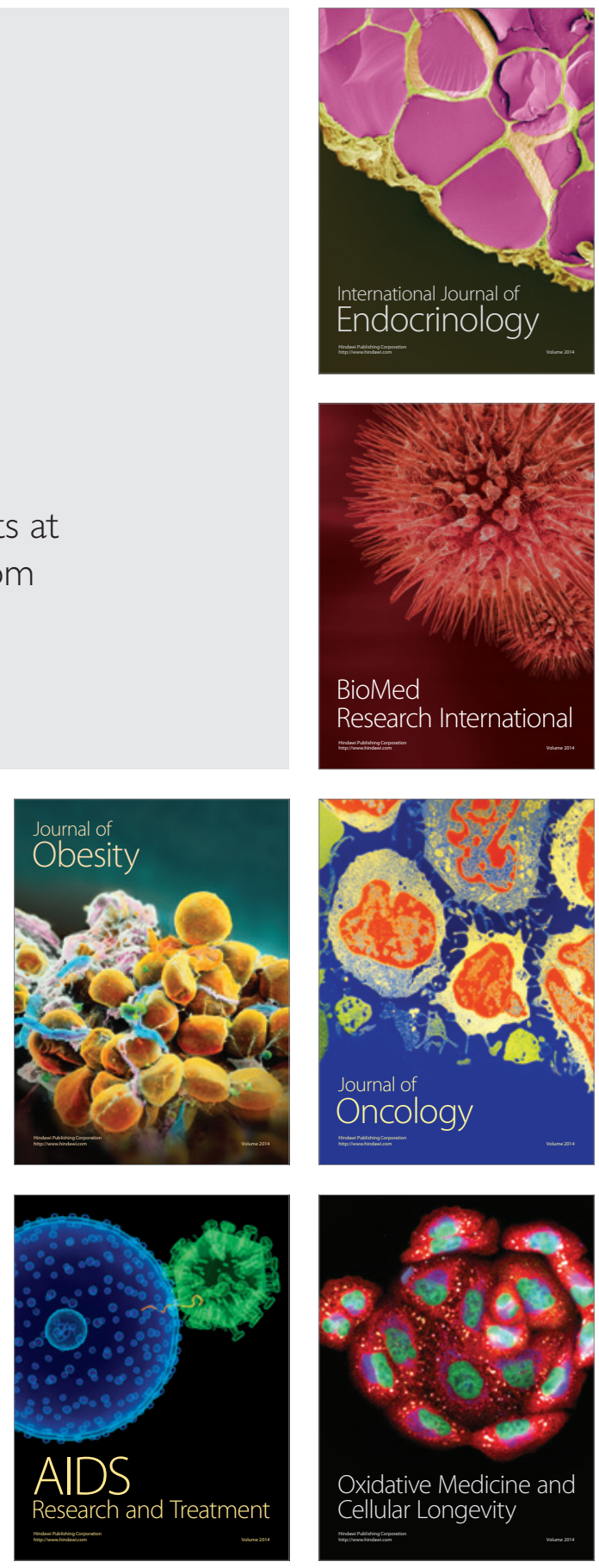